\title{
OUBLIER DESCARTES ? \\ LES PASSIONS DE L'ÂME ET LES THÉORIES MODERNES DES ÉMOTIONS
}

\author{
DENIS KAMBOUCHNER \\ Université Paris 1 Panthéon-Sorbonne
}

\section{DESCARTES FORGOTTEN? THE PASSIONS OF THE SOUL AND MODERN THEORIES OF EMOTIONS}

\begin{abstract}
The reference to Descartes in modern scientific literature on emotions is either missing or negative. And yet, one can show that the theories of emotions laid out by William James in the 1890' or by Antonio Damasio one century later are to a great extent neo-Cartesian: it is Descartes who first construed the passions of the soul as the expressions of a "body landscape" and of a bodily reaction to certain impressions or stimuli. One can also show that Descartes' account deals with the complexity of the affective life, and of the passion itself as a special kind of thought, in a more resolute way than a number of modern approaches do. On both planes, the disdain and caricature of the Cartesian legacy appear regrettable and unjustified.
\end{abstract}

Keywords: Emotions, Descartes, W. James, A. Damasio, Psychophysiology, Philosophy of Mind

Mots clés : émotions, Descartes, W. James, A. Damasio, psychophysiologie, philosophie de l'esprit

Les quelques remarques qui suivent appellent un mot de délimitation. Sur la nature des émotions, la littérature contemporaine est considérable, dans les domaines des neurosciences et de la philosophie de l'esprit, de la psychologie, de la psychanalyse même, et il ne pourra pas être question ici d'en restituer l'ampleur. À quelques exceptions près, cependant, cette littérature se meut dans un cercle du contemporain, et semble ignorer beaucoup ou presque tout de ce qui s'est écrit sur les émotions ou passions de l'âme avant William James, ou au mieux avant Darwin ; ou si elle ne l'ignore pas, elle ne s'y attarde pas non plus. C'est cette situation qui fera mon objet : l'héritage explicite apparaît fort mince, ce qui n'empêche pas que l'on puisse se mettre en quête d'un héritage caché, invisible ou dénié. En tout état de cause, il y a quelque sens à vouloir appréhender tout à la fois la relation et la distance historique et philosophique entre les perspectives adoptées aujourd'hui sur les émotions et celles qui ont été mises en œuvre à un moment que l'on s'accorde à désigner comme le départ de la pensée moderne. Je m'intéresserai en premier lieu à cette relation, puis plus succinctement à cette distance. 


\section{Damasio, cartésien malgré lui}

Toute une partie de la recherche contemporaine, qui étudie la formation des émotions dans le cerveau et dans le corps et leur fonction dans le comportement humain, répond à une inspiration bien plus cartésienne qu'elle ne le suppose ou ne le reconnait le plus souvent.

Le cas le plus notoire et, avouons-le, le plus caricatural est celui d'Antonio Damasio avec son Erreur de Descartes ${ }^{1}$. Ce cas est précisément trop notoire pour que l'on doive ici s'y attarder ${ }^{2}$. Rappellons seulement les thèses positives de l'ouvrage :

1) Loin d'avoir de manière générale l'effet perturbateur qu'on leur a attribué, l'expression et la perception des émotions sont intégrées aux mécanismes de la faculté de raisonnement ${ }^{3}$. En témoignent les lésions d'une certaine région du cerveau humain - le cortex préfrontal ventro-médian - qui perturbent de façon constante et très nette les deux types de processus à la fois 4 .

2) L'émotion n'est pas dans son essence une qualité mentale insaisissable, attachée à un objet ; elle " correspond à la perception directe d'un paysage particulier : celui du corps ${ }^{5}$ ». Ce « paysage » change d'instant en instant, mais il nous est continuellement présent ; mais comme sa perception se « juxtapose » à la perception et au souvenir de choses qui ne font pas partie du corps ${ }^{6}$, ces émotions finissent par être des "qualificatifs", qualifiers, pour ces autres choses.

3) Ainsi, la manière dont nous construisons (i. e. : nous représentons) le monde autour de nous, et dont est constitué l'élément de subjectivité qui entre en permanence dans nos expériences, n'a pas sa base ailleurs que dans notre organisme ${ }^{7}$. Certains chercheurs se sont demandé si des cerveaux placés au milieu d'une cuve dans des conditions électro-chimiques adéquates pourraient avoir les mêmes perceptions que nous ; mais non : il manquerait ici des interactions avec le corps qui sont indispensables et caractéristiques ${ }^{8}$.

Damasio présentait ces trois thèses comme directement contraires à celles de Descartes. En posant l'indépendance ontologique de l'esprit par rapport au corps, Descartes aurait affirmé l'indépendance fonctionnelle de la faculté de raisonner par rapport à la vie affective. Son erreur :

Avoir instauré une séparation abyssale entre le corps et l'esprit - entre, d'un côté, la matière corporelle, pourvue de grandeur et de dimensions, mue mécaniquement, divisible à l'infi-

1 Damasio, A. R. (1994) : Descartes' Error: Emotion, Reason and the Human Brain. New York: G. P. Putnam's Sons, Books (ci-après DE) ; trad. fr. (très défectueuse) : L'Erreur de Descartes. La raison des émotions. Paris : Odile Jacob, 1995 (ci-après ED).

2 J'ai abordé ce sujet dans «L'erreur de Damasio : la transition Descartes-Spinoza en psychophysiologie ». In Jaquet, Ch. - Sévérac, P. - Suhamy, A. : La théorie spinoziste des rapports corps/esprit et ses usages actuels. Paris : Hermann, pp. 199-215.

3 In Jaquet, Ch. - Sévérac, P. - Suhamy, A. : La théorie spinoziste des rapports corps/esprit et ses usages actuels. Paris : Hermann, pp. 199-215.

ED, p. 100 et pp. 233-235.

DE, pp. xv/11-12.

Ibid., p. xv/12.

Ibid., p. xvi/13.

Ibid., pp. 227-228, 286-287. 
ni, et de l'autre, l'étoffe mentale [mind stuff], sans grandeur, sans dimensions, impossible à mouvoir, indivisible ; avoir suggéré que le raisonnement, le jugement moral, la souffrance qui vient de la douleur physique ou le bouleversement émotionnel pouvaient avoir lieu indépendamment du corps. Et spécifiquement, avoir séparé les plus délicates opérations de l'esprit de la structure et du fonctionnement d'un organisme biologique 9 .

Cette " erreur de Descartes " n'est pas celle de tout le rationalisme classique, mais seulement celle d'une métaphysique dualiste qui a fait du penser et de la conscience de penser « les substrats réels de l'être ${ }^{10}$ ». Une telle métaphysique aura trouvé son contradicteur en Spinoza, dont la position, par contraste, apparaît remarquablement proche de l'inspiration actuelle des neurosciences :

Qu'est-ce que le conatus spinoziste en termes biologiques actuels ? C'est l'agrégat de dispositions contenues dans les circuits cérébraux qui, dès lors qu'elles sont enclenchées par des conditions internes ou environnementales, recherchent à la fois la survie et le bien-être ${ }^{11}$.

Du reste, Spinoza a défini de manière pertinente la joie et la tristesse comme perceptions de l'état interne du corps, et affirmé avec une égale pertinence l'unité de l'esprit et du corps, en posant l'esprit comme idée du corps humain ${ }^{12}$.

Le problème est que Damasio méconnaît ici entièrement la proximité ou même la solidarité foncière, par-delà la différence des axiomatiques, entre la problématique spinoziste des affects et l'analyse cartésienne des passions de l'âme. C'est aussi que de Descartes, il n'a pratiquement rien lu : à son propos, il ne fait que reproduire les stéréotypes en usage dans le monde scientifique, notamment anglo-saxon, et ne retient du grand traité de psychophysiologie que constituent les Passions de l'âme, que l'intéressante vérité suivante : «La domination des penchants animaux par la pensée, la raison et la volonté est ce qui fait de nous des êtres humains ${ }^{13}$ ".

C’est pourtant Descartes qui, après avoir rédigé une première version des Passions de l'âme, écrivait fin 1646 à son ami Chanut :

Il semble que vous inférez, de ce que j'ai étudié les passions, que je n'en dois plus avoir aucune; mais je vous dirai que tout au contraire, en les examinant, je les ai trouvées presque toutes bonnes, et tellement utiles à cette vie, que notre âme n'aurait pas sujet de vouloir demeurer jointe à son corps un seul moment, si elle ne les pouvait ressentir ${ }^{14}$.

Et s'agissant des passions, il est fort aisé de rétablir les points suivants :

9 Ibid., pp. 250-251 (ma traduction), 312.

10 Ibid., pp. 248, 311.

11 Damasio, A. R. (2003) : Looking for Spinoza: Joy, Sorrow and the Feeling Brain. Orlando Fla. : Harcourt; trad. fr. : Spinoza avait raison. Paris : Odile Jacob, 2003, p. 41.

12 Ibid., p. 211.

13 DE, p. 124 ; ED, p. 165.

14 Descartes à Chanut, $1^{\mathrm{er}}$ novembre 1646. In CEuvres de Descartes. (1964-1974). Éd. Adam-Tannery (ci-après AT), Paris : Vrin-CNRS, nouvelle présentation, t. IV, p. 538. Descartes (2014) : Correspondance. Éd. de J.-R. Armogathe (CEuvres complètes, t. VIII ; ci-après OC). Paris : Gallimard-Tel, vol. 2, p. 675. 
1) Dans leur définition cartésienne, les passions de l'âme sont en premier lieu des " perceptions qui dépendent des nerfs ${ }^{15}$ ", et qui expriment l'état interne du corps, et donc ont du rapport, pour parler comme Damasio, avec le « paysage corporel ». Bien entendu, elles ne sont pas que cela : ces pensées d'un type particulier qu'on appelle passions impliquent une forme de prise de position ou de mouvement à l'égard d'un objet qui est en général extérieur au corps propre. Mais Damasio lui-même admet tout-à-fait cette extension, avec la « juxtaposition ", à l'émotion elle-même, de perceptions et de souvenirs de choses autres que le corps.

2) Descartes admet divers cas d'excitation des passions. Celles-ci peuvent être excitées dans l'âme par le corps, du seul fait de son état interne (c'est un cas-limite) ${ }^{16}$. Elles peuvent l'être par l'âme qui d'elle-même pense à quelque chose ${ }^{17}$. Plus généralement, elles sont excitées par le surgissement d'un objet ou par un événement extérieur qui se trouvera perçu par l'âme à peu près en même temps qu'il fera effet sur le corps. Mais dans aucun de ces cas, l'émotion physiologique ne peut être dite sans fonction par rapport à l'âme. Dans le premier cas (origine purement somatique de la passion), lâme est renseignée sur l'état du corps et incitée à faire quelque chose qui se rapporte à cet état. Dans le second comme dans le troisième, où il y a représentation d'objet, une certaine pensée qu'elle a ou qu'elle peut avoir par elle-même s'agissant de cet objet acquiert un supplément de poids qui la rend plus considérable, notamment en tant qu'elle présente une action à accomplir. On dira certes que la motion passionnelle ne doit pas toujours être suivie, mais elle doit être toujours considérée.

3) De fait, il existe toute une thématisation cartésienne de "l'usage des passions ${ }^{18}$ ", qui met en relief la nécessaire contribution de celles-ci à la vie pratique. Lâme cartésienne ne doit certes pas suivre mécaniquement ses passions : elle doit en juger, et le cas échéant - mais seulement le cas échéant - s'efforcer d'agir selon un autre principe que celui que lui propose sa passion présente. Le fait que les motions passionnelles soient à mettre en délibération ne constitue en aucune manière une proposition philosophiquement scandaleuse ou contestable, étant donné d'abord que personne ne suppose qu'à quelque moment que ce soit, l'affect (émotion ou passion) présent dans l'esprit soit unique et simple. Mais il faut ajouter qu'en coordonnées cartésiennes, la réflexion sur la passion présente, qui aboutit le cas échéant à en désamorcer la dynamique, n’est pas elle-même une opération purement intellectuelle au sens où elle serait radicalement inaffective. Cette réflexion sur les passions met en œuvre des émotions d'un genre particulier, que Descartes appelle les émotions intérieures de l'âme : celle dans lesquelles l'âme consomme l'effet que cela lui fait d'éprouver telle ou telle passion ${ }^{19}$. On peut assurément discuter (nous y reviendrons ci-après) des caractères de ces émotions intérieures, ici regardées comme exemptes de toute dimension physiologique, mais cette discussion ne doit pas masquer l'essentiel : l'étendue cartésienne de la fonction affective, et son indissociabilité absolue d'avec l'exercice du raisonnement pratique.

15 Voir Les Passions de l’âme (ci-après Passions), art. 25 à 27.

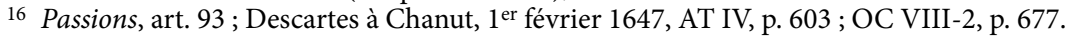

17 Ibid.

18 Voir Passions, art. 137 à 143.

19 Voir Passions, art. 147 et 148, et mes remarques dans L'Homme des passions. (1995). Paris : Albin Michel, t. II, pp. 169-196. 
Réserve faite des différences manifestes et considérables entre le cerveau de Descartes et celui de Damasio, on devrait donc pouvoir admettre que les vues développées par Damasio sur la fonction des émotions sont beaucoup plus néocartésiennes qu'il ne pense.

\section{Émotions jamesiennes et passions cartésiennes}

Reportons-nous maintenant un siècle en arrière, du côté de cet ouvrage fondateur de la psychologie moderne que sont les Principles of Psychology de William James $(1890)^{20}$. Dans le célèbre chapitre XXV, que James consacre aux émotions, l'on peut vérifier une continuité du même ordre, et qui pas plus que plus tard chez Damasio n'est explicite.

James distingue entre les émotions fortes (coarser emotions), colère, peur, amour, haine, joie, peine (tristesse), honte, orgueil, qui sont fortes y compris dans leur aspect physique, et les émotions plus subtiles (subtler) qui sont les sentiments moraux, intellectuels et esthétiques, avec une réaction physique d'ordinaire beaucoup moins forte. James écrit (c'est sa thèse centrale) :

L'idée que nous nous faisons naturellement de ces émotions fortes est que la perception mentale d'un fait donné excite dans l'esprit l'affection qu'on appelle émotion, et que cet état mental suscite une expression corporelle. Ma théorie est au contraire la suivante : les changements corporels suivent directement la perception du fait stimulant, et notre sentiment de ces changements au moment où ils surviennent est l'émotion elle-même (our feeling of the same changes as they occur IS the emotion $)^{21}$.

Selon cette vue, et la formule est célèbre, « il est plus rationnel de poser que nous sommes désolés parce que nous pleurons, en colère parce que nous frappons, effrayés parce que nous tremblons ", que l'inverse ${ }^{22}$.

La nécessité de ce renversement est corroborée par les cas pathologiques d'émotion sans objet : ainsi avec cette " peur morbide » qu'engendre, sans rien qui la motive, un syndrome formé de troubles de la respiration, de palpitations cardiaques et de ce changement de l'épigastre (du creux de l'estomac) caractéristique de l' " anxiété précordiale ». On peut aussi évoquer le rapport entre l'attention volontaire aux manifestations de l'émotion en soi, et la production ou le renforcement de cette émotion elle-même. L'émotion, écrit James, n'est ici rien d'autre que le sentiment d'un état corporel, et sa cause est purement corporelle ; elle intègre seulement le sentiment, vif ou confus, d'un très grand nombre de changements corporels, qui font toutes les nuances des émotions. Ces nuances sont éminemment changeantes, différentes d'un individu à l'autre et susceptibles d'une variété illimitée - raison pour laquelle les efforts de classification n'auront qu'une signification limitée. L'argument central («the vital point of my whole theory») est ici que :

${ }^{20}$ James, W. (1890) : Principles of Psychology (ci-après PP). New York: Henry Holt and Company, 2 vol. ; réimpression New York : Dover, 1950.

21 PP II, p. 449.

22 Ibid., p. 450. 
si nous imaginons une forte émotion, puis tentons d'abstraire de la conscience que nous en avons toutes les sensations de ses symptômes corporels, nous constatons qu'il ne reste rien derrière, aucune 'étoffe mentale' (mind-stuff) dont l'émotion pourrait être constituée ${ }^{23}$.

L'émotion dissociée de toute sensation corporelle est inconcevable ; une émotion humaine désincarnée est un pur néant.

Ces effets corporels si particuliers et si divers qui se remarquent dans les émotions s'effectuent sur un mode réflexe ${ }^{24}$ et correspondent à des mécanismes préorganisés ${ }^{25}$. À la différence de la réaction instinctive (motrice), la réaction émotionnelle s'achève dans le corps du sujet ; mais une simple imagination peut suffire à la déclencher.

La question est : à quoi ces phénomènes peuvent-ils tenir? James évoque ici deux principes. (1) Certains mouvements sont des répétitions affaiblies de mouvements qui étaient, sous leur forme initiale, utiles au sujet, ou bien de mouvements qui étaient, en d'autres circonstances, des concomitants physiologiquement nécessaires des mouvements utiles. Ainsi, les troubles respiratoires qu'on observe dans la colère et dans la peur seront des réminiscences organiques des halètements d'un homme dans l'effort du combat, ou de l'essoufflement de celui qui s'enfuit précipitamment. Ce sont des amorces ou des répétitions de réactions qui ont été utiles dans une confrontation plus violente avec l'objet qui suscite l'émotion. (2) Des stimuli perçus comme analogues excitent des réactions similaires. C'est ainsi que Wundt et Piderit ont évoqué des mouvements gustatifs comme symbolisant beaucoup de nos réactions aux problèmes moraux ${ }^{26}$.

Or, ces deux points sont parfaitement classiques. Au cœur de l'apport cartésien, on trouve la relation indissociable entre la passion de l'âme, le mouvement de la glande pinéale qui est au milieu du cerveau et constitue le "siège principal de l'âme ${ }^{27}$ ", et enfin la stimulation du petit nerf qui descend du cerveau vers le cœur (nerf de la sixième paire), en même temps que celle de toutes sortes de nerfs insérés dans les parties intérieures du corps et dans les muscles. Nous l'avons vu, la passion étant la répercussion dans l'âme d'une émotion physiologique, elle doit être - ou être accompagnée de - la perception confuse de toutes ces modifications. Sous ce rapport, il s'agit d'une perception globale de l'état du corps, à distinguer de la perception particulière de ce qui affecte telle ou telle partie du corps.

Il y a bien un effort cartésien pour établir une typologie des manifestations physiques de toutes sortes et pour établir de grands schémas de modifications correspondant à ce que Descartes appelle les « passions primitives ${ }^{28}$ ». Mais il est clair, comme Descartes l'indique à la princesse Élisabeth en mai 1646, que l'on a toujours affaire à plusieurs passions ensemble ${ }^{29}$, et que les formes physiologiques de l'émotion passionnelle varient en fonction des individus et des moments. Cette individuation des passions sera encore plus fortement marquée chez Spinoza ${ }^{30}$.

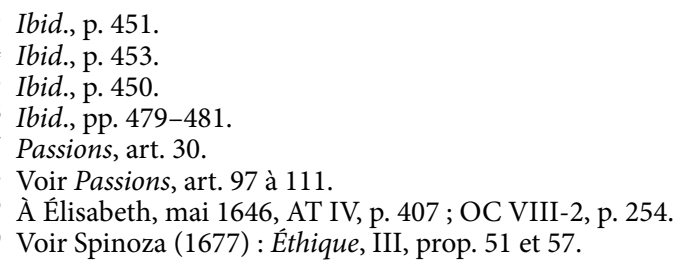


Second point : les passions sont, chez Descartes comme chez Hobbes ${ }^{31}$, des commencements de réactions motrices qui ont d'abord des objets corporels, mais dont les objets dans la vie adulte sont souvent très différents, bien qu'en relation analogique avec les premiers. Pour Descartes, les premières passions de notre vie sont celles que notre âme nouvellement unie à notre corps a éprouvées dans le ventre de nos mères ${ }^{32}:$ l'amour, par exemple, du fait que survenait un aliment particulièrement convenable à l'entretien de la chaleur cardiaque, ou la tristesse, du fait d'une insuffisante alimentation de cette chaleur. Mais dans les phases ultérieures de la vie, c'est toute sorte de bon aliment, y compris de notre âme, ou tout défaut d'un pareil aliment qui déclenchera de pareilles passions.

Pour l'essentiel, les deux points marqués par James sont donc déjà cartésiens, et d'ailleurs partagés par d'autres auteurs du XVII e siècle. Pourtant, James présente sa thèse d'après laquelle l'émotion suit de modifications corporelles ou plutôt coïncide avec elles plutôt qu'elle ne les provoque comme devant susciter l'incrédulité33. Et pour la fonction de ces modifications corporelles, ce n'est que très récemment, ajoute-t-il, que la question a été posée. Cette assertion s'accompagne de références à Spencer, Principles of Psychology, 1855 ; Piterit, Wissenschaftliches System der Mimik und Physiognomik, 1867 ; Darwin, The Expression of the Emotion in Man and Animals, 1872 ; Wundt, Grundzüge der Physiologischen Psychologie, 1874 ; Mantegazza, La physionomie et l'expression des sentiments, 1885. Aucune allusion n'est faite aux auteurs classiques.

Dans tous les Principles, on trouve quatre références à Descartes dans le tome I ${ }^{34}$ (sur les animaux-machines, la chose qui pense, l'union de l'âme à la glande pinéale, la substantialité de l'âme) ; trois références à Hobbes, dans le même tome I, chapitre sur l'association $^{35}$; une référence à Spinoza à propos de la perception de la réalité, avec citation du scolie final de la Seconde partie de l'Éthique (II, proposition 49) ${ }^{36}$; une citation de Malebranche (Entretiens sur la métaphysique et la religion, III), à propos de la confusion des impressions sensibles, dans une note du chapitre sur la sensation ${ }^{37}$. Mais au chapitre des émotions, Descartes n'est absolument pas cité, et on lira seulement dans l'abrégé des Principles, le Briefer Course de 1892, que la littérature purement descriptive sur les émotions, « depuis Descartes », est l'une des parties les plus ennuyeuses de la psychologie ${ }^{38}$.

Les citations de Hobbes, de Malebranche et de Spinoza, sans compter la plus grande place faite à Berkeley, Locke ou Hume, témoignent de l'ample culture classique qui est celle de James. Il y a donc une manière d'énigme dans la non-reconnaissance de la continuité dont il s'agit. Mais sans doute la chose s'explique-t-elle, comme dans le cas de Damasio, par la représentation d'un Descartes beaucoup plus nettement dualiste qu'il ne l'est en réalité. James n'ignore pas l'effort de Descartes pour expliquer l'ensemble des actions des animaux par la disposition de leurs organes, et le cas échéant par celle de leur cerveau. Un passage du tome I l'atteste : «Il revient à Descartes d'avoir été le premier

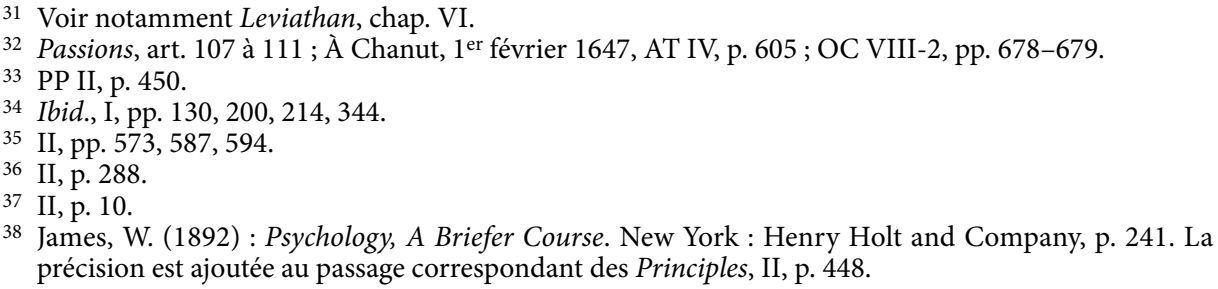


à concevoir un mécanisme nerveux entièrement autosuffisant, susceptible de nous faire effectuer des actions compliquées et apparemment intelligentes ${ }^{39}$. »

Mais dans la même page où Descartes est crédité d'avoir conçu un arc sensori-moteur complet, le reproche suit immédiatement : «Par une restriction singulièrement arbitraire, Descartes s'est arrêté court devant l'homme, et, en tenant que dans les bêtes tout était machinerie nerveuse, a considéré que les actes les plus élevés de l'homme relevaient de l'action de son âme rationnelle ${ }^{40}$. "

Il est certain que pour Descartes, la conduite de la vie humaine aussi bien que la recherche de la vérité relèvent de facultés qui ne sont pas dans les bêtes ; que l'âme, nous l'avons dit, a le pouvoir d'exciter en elle-même certaines passions, par la disposition qu'elle a d'une partie au moins de ses pensées ; et qu'elle devrait toujours contrôler et maîtriser ses passions par l'usage qu'elle a de sa raison. Il n'empêche que Les Passions de l'âme ont poussé très loin l'étendue et la sophistication du pouvoir que le corps, avec les passions, exerce sur l'âme. Dans les passions, c'est toute une mémoire corporelle, inscrite dans les plis du cerveau, qui s'exprime et qui définit pour l'âme elle-même le caractère de l'objet ou de la circonstance présente ; c'est donc aussi le corps qui précède l'âme dans la définition d'une réaction à cette circonstance, et cela, tantôt de manière précipitée, tantôt de manière salutaire, lorsque la conservation du corps et donc du composé de l'âme et du corps est en jeu. C’est ce qui conduit Descartes à écrire ceci qui m'a toujours paru très audacieux : "L'utilité de toutes les passions ne consiste qu'en ce qu'elles fortifient et font durer en l'âme des pensées, lesquelles il est bon qu'elle conserve, et qui pourraient facilement sans cela en être effacées ${ }^{41}$. »

Il est vrai qu'il ajoute aussitôt : « [...] Comme aussi tout le mal qu'elles peuvent causer, consiste en ce qu'elles fortifient et conservent ces pensées plus qu'il n'est besoin ; ou bien qu'elles en fortifient et conservent d'autres, auxquelles il n'est pas bon de s'arrêter ${ }^{42}$. »

Mais Dieu aurait mal fait les choses, dans ce que Descartes appelle l'institution de la nature, si le mal l'emportait sur le bien. Et comme il sera dit à la fin du traité, "nous voyons que [les passions] sont toutes bonnes de leur nature, et que nous n'avons rien à éviter que leurs mauvais usages ou leurs excès ${ }^{43}$ ".

Entre l'approche cartésienne et l'approche jamesienne, on peut assurément relever un certain nombre de différences, comme l'a fait dans une substantielle étude un interprète très autorisé, Gary Hatfield ${ }^{44}$.

Descartes et James, souligne Hatfield, proposent certes tous les deux des body-first theories : ils considèrent tous deux la peur et la fuite comme deux effets distincts d'un système corporel visant à produire des comportements appropriés aux situations. Tous deux distinguent entre les premières émotions de l'enfant et les émotions développées de l'adulte, qui reflètent l'expérience passée et les apprentissages, et sont excitées par des facteurs cognitifs complexes ${ }^{45}$. Mais, ajoute Hatfield, les deux théories diffèrent en ce que,

39 PP I, p. 130.

40 Ibid.

41 Passions, art. 74.

42 Ibid.

43 Passions, art. 211.

44 Hatfield, G. (2007) : "Did Descartes have a Jamesian Theory of the Emotions? », Philosophical Psychology, vol. 20, 4, pp. 413-440.

45 Ibid., p. 414. 
chez James, l'appréciation cognitive de la situation ne fait pas partie du contenu propre des émotions. La théorie jamesienne est, dira-t-on dans les termes d'aujourd'hui, non-cognitive : les émotions sont des perceptions (perceptions des changements internes du corps, ou de son état) plutôt que des pensées au sens courant du mot ${ }^{46}$. Ce n'est qu'avec certains développements subséquents que nous pouvons faire le rapport entre nos émotions et des situations objectives, et leur accorder ainsi une signification cognitive. Du même fait, chez James, l'état émotionnel ne serait pas en lui-même ou originellement motivant (motivating, motivational), même si les émotions développées, réfléchies, connectées peuvent revêtir un aspect motivationnel.

Au contraire, souligne encore Hatfield, les passions chez Descartes sont des sentiments (feelings) que le sujet pensant (the self) reconnaît comme les siens propres ${ }^{47}$. Ce sont en effet « des perceptions, des sentiments ou des émotions qu'on rapporte particulièrement à [l'âme $]^{48}$ ", à la différence des « autres sentiments, qu'on rapporte les uns aux objets extérieurs, [...] les autres à notre corps [... ${ }^{49}$ ". Aussi, quoique les passions soient causées par des conditions corporelles, elles ne sont pas des perceptions de ces conditions internes. Elles représentent le « caractère de la situation », et c'est par là qu'elles ont une action sur la volonté ${ }^{50}$.

Il n'est pourtant pas certain que les choses soient aussi tranchées, avec d'un côté, chez Descartes, une passion-représentation, et de l'autre, chez James, une passion-sensation. James dit bien que comme états internes, les émotions ne sont pas descriptibles, et que tout ce qu'on peut noter, ce sont leurs rapports avec les objets qui les suscitent et avec les réactions qu'ils provoquent ${ }^{51}$. Et d'autant que le même James admet une connexion très générale entre l'émotion et une réaction instinctive qui a cessé d'être aveugle, il apparaît difficile de dissocier le feeling émotionnel d'une sorte de direction intentionnelle. À cet égard, la formule : " our feeling of the same bodily changes as they occur IS the emotion ${ }^{52}$ » est loin d'épuiser la question.

De la même manière, chez Descartes, il n'y a absolument aucun moyen de séparer la dimension sensitive de l'émotion de sa dimension intentionnelle, ni par conséquent de la perception d'un certain état de chose. C'est plutôt cette superposition, cette surimpression, ou plutôt cette pluridimensionnalité ou compacité qui caractérise le phénomène de la passion. La passion n'est représentation de situation que lestée par le corps, par la conscience d'une intention du corps marquée sur le mode le plus sensitif.

\section{Les deux facteurs de la distance}

Venons-en aux indices de distance. Il y a deux points principaux à considérer.

En premier lieu, toute une partie des discussions contemporaines sur les émotions s'écarte de l’inspiration cartésienne, précisément par son exigence de catégorisation.

46 PP I, pp. 221-222.

47 Article cité, p. 423.

48 Passions, art. 27.

49 Ibid., art. 29.

50 Article cité, p. 426.

51 Briefer course, ch. 18, p. 373.

52 PP II, p. 449. 
Nous ne sommes plus cette fois chez les neurobiologistes, mais chez les philosophes de l'esprit. Ceux-ci se demandent non seulement quelle est la fonction des émotions, mais ce qu'elles sont en tant qu'elles prennent place dans l'esprit. Ainsi, il existe une théorie dite perceptuelle des émotions, d'après laquelle celles-ci constituent des perceptions d'un type spécifique : ce ne sont pas simples sensations corporelles (car alors elles n'auraient pas à proprement parler d'objet externe, alors que la peur est, par exemple, peur du loup) ; ce ne sont pas non plus proprement des jugements, car alors elles seraient toujours liées à des concepts, et il ne faudrait pas parler d'émotions des tout jeunes enfants. Selon cette théorie " perceptuelle », telle que résumée par exemple par Christine Tappolet dans un article très clair ${ }^{53}$, les émotions sont des perceptions de valeurs positives ou négatives, et leur rôle crucial tiendra justement au fait qu'elles nous rendent conscients de la valeur des choses. C'est en tant que perceptions de valeurs qu'il faudra les rapprocher des perceptions sensorielles, sous trois rapports : leur caractère conscient, le fait qu'elles sont en général causées par les choses qui nous environnent, et le fait qu'elles ne sont pas directement soumises à la volonté. Mais dans ce cas, comment une émotion peut-elle être mauvaise - alors qu'on ne dit pas, dans le même sens, d'une perception sensorielle qu'elle est mauvaise?

Par rapport à ce genre de discussion, l'historien de la philosophie pourra conserver le sentiment que les problèmes sont posés dans un cadre trop étroit, et avec peu de bénéfice par rapport à la subtilité des formules que l'on trouve chez les auteurs classiques. Tous (Descartes, Hobbes, Malebranche, Spinoza et quelques autres) donnent certes aux passions, sauf exception, un objet qualifié, et chez tous, les passions ont en nous pour effet sinon de produire cette qualification, du moins de la renforcer. Ainsi, chez Descartes, l'amour, incitant l'âme « à se joindre de volonté aux objets qui paraissent lui être convenables ${ }^{54}$ ", les lui représente instamment comme convenables. Mais ni chez Descartes ni chez les autres classiques, l'accent n'est placé sur cette spécification de l'objet des passions, qui se déclinerait au risque de la monotonie : l'amour/l'aimable, la haine/le haïssable, le désir/le désirable, la joie/le réjouissant, etc. Il est au contraire essentiel que la qualité ou valeur attribuée à l'objet des passions soit mentionnée parmi d’autres énoncés ou clauses relatives à ces passions. Et de fait, autant il est légitime de dire de la passion qu'elle a pour effet de renforcer en nous la perception de l'objet comme ceci ou cela, autant il est improbable que l'on puisse la définir en tout et pour tout comme cette perception. Nous ne devons jamais oublier, s'agissant de la passion en tant qu'elle appartient à l'âme, que nous ne savons pas proprement en quoi elle consiste, et qu'un certain aspect d'obscurité et de confusion en est constitutif.

Second point : Descartes distinguait entre les passions et des « émotions intérieures de l'âme ", ou des émotions intellectuelles (d'amour, de haine, de joie et de tristesse) qui suivent immédiatement des jugements que l'âme effectue et sont exclusives de dimension physiologique 55 . C'est là une tout autre distinction que celle que James a proposée entre les émotions fortes (coarser) et les émotions plus subtiles (subtler), en niant précisément -

53 Tappolet, C. (2011) : «Les mauvaises émotions ». In Tappolet, C. - Teroni, F. - Konzelmann Ziv, A. : Les ombres de l'âme. Penser les émotions négatives. Genève : Éd. Markus Heller, pp. 37-51.

54 Passions, art. 79.

55 Principes de la philosophie, IV, art. 190 ; Passions, art. 29, 79, 91, 92, 147, 148, 187 ; À Chanut, $1^{\text {er février }}$ 1647, AT IV, pp. 601-603; OC VIII-2, pp. 676-678. 
et tous les penseurs modernes à sa suite - qu'il y ait un sens à parler d'une émotion sans dimension physiologique et sensorielle.

C'est bien évidemment dans cette distinction entre passions et émotions intellectuelles que se marque le « dualisme » cartésien, au prix du reste d'une énigme persistante concernant la manière dont l'âme peut se trouver émue sans aucune marque sensible de cette émotion. Cette énigme ne peut se réduire que si l’on considère que les émotions dont il s'agit sont en pratique indissociables des passions, qu'elles déterminent, qu'elles suivent ou qu'elles prennent pour objets (ceci exclusivement en tant qu' « émotions intérieures de l'âme »). Elles ne sont donc en réalité jamais seules dans l'âme, et ce n'est que par opposition au caractère très sensible de la passion qu'elles apparaissent dépourvues de cette dimension physiologique ${ }^{56}$.

Quoi qu'il en soit, la fonction de cette distinction doit être bien mesurée : elle signifie qu'il existe des émotions de second degré, et qu'il est essentiel qu'il en existe ; que, sur le plan de l'affectivité comme sur celui du jugement, nous ne sommes presque jamais tout d'une pièce ; que nous réagissons à nos propres passions et pouvons mettre une certaine distance entre elles et nous, distance qui peut s'interpréter comme une distance entre deux " nous» (entre deux « moi » ou " soi »). Avec cette sorte d'objectivation s'ouvrent toutes sortes de possibilités de pensée, de langage et d'action. Celles-ci, notamment la complexité intrinsèque de toute déclaration de passion, sont bien connues des psychologues, des psychanalystes, des experts en littérature et de quelques philosophes. Les écrivains et moralistes de l'âge classique, tels Racine ou La Rochefoucauld, les ont profondément méditées. Descartes n'a pas constitué seul l'ample mémoire dans laquelle nous pouvons puiser, mais avec sa concision propre il y a sans nul doute contribué pour une part notable, et à cet égard, un fil sinueux mais solide court de son œuvre jusqu'à celle de Proust. La question décisive consiste à savoir si les théories des émotions auxquelles nous avons aujourd'hui affaire sont capables de reconnaître et de prendre en charge cette complexité. Si la réponse devait être négative, il ne faudrait pas seulement parler d'oubli, mais de régression.

\section{BIBLIOGRAPHIE}

Damasio, A. R. (1994) : Descartes' Error : Emotion, Reason and the Human Brain. New York : G. P. Putnam's Sons Books ; trad. fr.: L'Erreur de Descartes. La raison des émotions, Paris : Odile Jacob, 1995.

Damasio, A. R. (2003) : Looking for Spinoza : Joy, Sorrow and the Feeling Brain. Orlando, Fla. : Harcourt; trad. fr. : Spinoza avait raison. Paris : Odile Jacob, 2003.

Descartes (1964-1974) : CEuvres. Éd. par Ch. Adam et P. Tannery. Paris : Vrin-CNRS, nouvelle présentation, $11 \mathrm{vol}$.

Descartes (2014). Correspondance. Éd. de J.-R. Armogathe. Paris : Gallimard-Tel, 2 vol. (CEuvres complètes, VIII, t. 1 et 2).

Hatfield, G. (2007) : «Did Descartes have a Jamesian Theory of the Emotions? », Philosophical Psychology, 20, 4, pp. 413-440.

James, W. (1890) : Principles of Psychology. New York: Henry Holt and Company, 1890, 2 vol. ; réimpression New York : Dover, 1950.

56 J'ai traité en détail de ce point dans L'Homme des passions, op. cit., t. I, pp. 345-367. 
James, W. (1892) : Psychology, A Briefer Course. New York : Henry Holt and Company. Trad. fr. par N. Féron, Précis de psychologie, Paris : Les Empêcheurs de penser en rond, 2003.

Kambouchner, D. (1995) : L'Homme des passions. Commentaires sur Descartes. Paris : Albin Michel, 2 vol.

Kambouchner, D. (2009) : "L'erreur de Damasio : la transition Descartes-Spinoza en psychophysiologie ». In Jaquet, Ch. - Sévérac, P. - Suhamy, A. : La théorie spinoziste des rapports corps/esprit et ses usages actuels. Paris : Hermann, pp. 199-215.

Kambouchner, D. (2015) : Descartes n'a pas dit. Paris : Les Belles-Lettres.

Spinoza (1925) : Éthique. In Spinoza : Opera. Éd. de C. Gebhardt. Heidelberg : Winters ; rééd. Olms, 1972, vol. III.

Tappolet, C. (2011) : « Les mauvaises émotions ». In Tappolet, C. - Teroni, F. - Konzelmann Ziv, A. : Les ombres de l'âme. Penser les émotions négatives. Genève : Éd. Markus Heller, pp. 37-51.

\section{Denis Kambouchner}

Centre de Philosophie Contemporaine de la Sorbonne

Université Paris 1 Panthéon-Sorbonne

17 rue de la Sorbonne - F 75005 Paris

denis.kambouchner@univ-paris1.fr 